\title{
Logical and Relational Learning
}

\author{
Luc De Raedt \\ Department of Computer Science, Katholieke Universiteit Leuven \\ Celestijnenlaan 200 A B-3001, Heverlee, Belgium \\ luc.deraedt@cs.kuleuven.be
}

I use the term logical and relational learning (LRL) to refer to the subfield of machine learning and data mining that is concerned with learning in expressive logical or relational representations. It is the union of inductive logic programming, (statistical) relational learning and multi-relational data mining and constitutes a general class of techniques and methodology for learning from structured data (such as graphs, networks, relational databases) and background knowledge. During the course of its existence, logical and relational learning has changed dramatically. Whereas early work was mainly concerned with logical issues (and even program synthesis from examples), in the 90s its focus was on the discovery of new and interpretable knowledge from structured data, often in the form of rules or patterns. Since then the range of tasks to which logical and relational learning has been applied has significantly broadened and now covers almost all machine learning problems and settings. Today, there exist logical and relational learning methods for reinforcement learning, statistical learning, distance- and kernel-based learning in addition to traditional symbolic machine learning approaches. At the same time, logical and relational learning problems are appearing everywhere. Advances in intelligent systems are enabling the generation of high-level symbolic and structured data in a wide variety of domains, including the semantic web, robotics, vision, social networks, and the life sciences, which in turn raises new challenges and opportunities for logical and relational learning. These developments have led to a new view on logical and relational learning and its role in machine learning and artificial intelligence. In this talk, I shall reflect on this view by identifying some of the lessons learned in logical and relational learning and formulating some challenges for future developments.

\section{Reference}

1. De Raedt, L.: Logical and Relational Learning. Springer, Heidelberg (in press) 